\title{
Sub-resolution Axial Localization of Nanoparticles in Fluorescence Microscopy
}

\author{
François Aguet, Dimitri Van De Ville, and Michael Unser \\ Biomedical Imaging Group \\ Ecole Polytechnique Fédérale de Lausanne, CH-1015 Lausanne, Switzerland
}

\begin{abstract}
We propose a method for sub-resolution axial localization of particles in fluorescence microscopy, based on maximum-likelihood estimation. Given acquisitions of a defocused fluorescent particle, we are can estimate its axial position within nanometer range precision.
\end{abstract}

\section{Introduction}

In recent years single particle tracking has significantly contributed to the study of intracellular molecular dynamics [2]. Particle tracking primarily consists of localizing particles in three dimensions (3-D) from a number of images acquired by fluorescence microscopy. While many approaches have been proposed for the lateral (i.e., x-y) localization of particles from acquisitions that are (almost) in focus [3], far fewer methods currently exist for the precise determination of a particle's axial (i.e., z) position. Since the acquisition of high-resolution z-stacks is inefficient and impractical, axial localization methods try to estimate the position using few acquisitions of the particle. The difficulty of this task comes from the complexity of the diffraction patterns that appear as the particle moves out of focus. To avoid complex models, current axial localization methods often rely on empirical approaches [4].

Under optimal conditions, the spot of light that one observes when viewing a sub-resolution fluorescent particle with a microscope corresponds to the diffraction-limited point spread function (PSF) of the microscope. While most methods for tracking and localizing single particles try to exploit this property, they often make use of a simplified diffraction model. The principal difficulty with respect to axial localization is the non-stationarity of the PSF along the optical axis; if there is a difference in the refractive indices of the specimen and immersion medium, the PSF may vary depending on the particle's depth within the specimen, which is a frequently occurring situation in practice. In this paper we propose an algorithm based on maximum-likelihood estimation that employs an accurate PSF model to iteratively determine a particle's axial position. We describe an image formation model, propose a theoretical lower bound on the attainable localization precision, and show how our algorithm is derived. Finally, we demonstrate its efficiency by showing the correspondence with the theoretical lower bound.

\section{Image formation and acquisition}

The essential basis for an axial localization approach is an accurate model of the image formation process, which describes how photons emitted by the particle are diffracted in the microscope, and how the photons exiting the microscope contribute to the digital image generated by the CCD camera mounted on the microscope. An image formation model that correctly takes into the account the non-stationarity of the PSF along the optical axis is crucial, since a wrongfully predicted PSF would result in a misestimation of the particle's position. We assume a general formulation of a 3-D PSF that is non-stationary with respect to the optical axis, given by $P S F\left(x-x_{p}, y-y_{p}, z \mid z_{p}\right)$, where $\left(x_{p}, y_{p}, z_{p}\right)$ are the coordinates of the particle, and where $(x, y, z)$ is the point where the PSF is measured (see Fig. 1-a). For the sake of simplicity, we shall express all coordinates in object space, including the pixel coordinates of acquisitions (as if acquisitions were demagnified and projected onto the focal plane).

Due to the nature of the specimen (i.e., diffusiveness, autofluorescence) and the type of image acquisition system used, noise from a variety of sources contributes to the recorded images of fluorescent particles. The dominant source is called photon (or shot) noise and results from statistical variation in the arrival rate of photons on the CCD. The nature of this variation implies that the recorded signal (photon count) at a given pixel on the CCD follows a Poisson distribution. Note that the effect of photon noise increases as the energy of the photon-emitting source decreases. In our image formation model, the photon count at a given pixel on the CCD follows a Poisson distribution whose mean is proportional to the intensity predicted by the PSF.

The probability of observing $q$ photons emitted by a particle located in $\left(0,0, z_{p}\right)$ at a point $(x, y)$ in the focal plane positioned at $z_{n}$ is then given by

$$
P\left(q\left(x, y, z_{n} \mid z_{p}\right)\right)=\frac{e^{-\bar{q}\left(x, y, z_{n} \mid z_{p}\right)} \bar{q}\left(x, y, z_{n} \mid z_{p}\right)^{q\left(x, y, z_{n} \mid z_{p}\right)}}{q\left(x, y, z_{n} \mid z_{p}\right) !},
$$


where $\bar{q}\left(x, y, z_{n} \mid z_{p}\right)$ is the expected number of photons. It is proportional to the intensity given by the PSF, such that

$$
\bar{q}\left(x, y, z_{n} \mid z_{p}\right)=c \operatorname{PSF}\left(x, y, z_{n} \mid z_{p}\right),
$$

where $c$ is a conversion factor that depends on the energy of the particle. The data from which we estimate the particle's position consists of $N$ acquisitions, which can be taken at different focal distances $z_{n}, n=1, \ldots, N$ (a higher number of acquisitions should obviously improve the estimation).

\section{A lower bound on the estimation precision}

The theoretical lower bound on the variance of any unbiased estimator is given by the Cramér-Rao bound (CRB). Expressing the CRB for our image acquisition model with respect to a particle situated at the axial position $z_{p}$ and simplifying the result yields

$$
\begin{aligned}
\operatorname{Var}\left(\hat{z}_{p}\right) & \geq 1 /-E\left[\frac{\partial^{2}}{\partial z_{p}^{2}} \ln \prod_{n=1}^{N} \prod_{(x, y) \in \mathcal{S}} P\left(\bar{q}\left(x, y, z_{n} \mid z_{p}\right)\right)\right] \\
& =1 / \sum_{n=1}^{N} \sum_{(x, y) \in \mathcal{S}} \bar{q}\left(x, y, z_{n} \mid z_{p}\right)^{-1}\left(\frac{\partial}{\partial z_{p}} \bar{q}\left(x, y, z_{n} \mid z_{p}\right)\right)^{2},
\end{aligned}
$$

where $\hat{z}_{p}$ is an unbiased estimator of the axial position $z_{p}$, and $\mathcal{S}$ is the set of pixels on the CCD (where an individual pixel has the coordinates $\left(x, y, z_{n}\right)$ ). The derivative $\frac{\partial}{\partial z_{p}} \bar{q}$ is an analytical expression obtained from the PSF model. This result gives us the fundamental limit on the precision that can be attained when estimating the position of a particle. To simplify the notation, we shall omit the arguments $\left(x, y, z_{n} \mid z_{p}\right)$ from this point on.

\section{Maximum-likelihood estimation algorithm}

An estimator for the axial position of a particle can be obtained by maximizing the joint likelihood; thus we derive with respect to $z_{p}$ :

$$
\frac{\partial}{\partial z_{p}} \ln \prod_{n=1}^{N} \prod_{(x, y) \in \mathcal{S}} P(q)=\sum_{n=1}^{N} \sum_{(x, y) \in \mathcal{S}}\left(\frac{\partial \bar{q}}{\partial z_{p}} \frac{q}{\bar{q}}-\frac{\partial \bar{q}}{\partial z_{p}}\right) \equiv 0 .
$$

For a realistic PSF model, it is not possible to solve directly for the estimated position $\hat{z}_{p}$. Therefore, we linearize the PSF model around the current estimate using the first-order Taylor approximation:

$$
\begin{aligned}
\frac{\partial}{\partial z_{p}} \ln \prod_{n=1}^{N} \prod_{(x, y) \in \mathcal{S}} P(q) & \approx \sum_{n=1}^{N} \sum_{(x, y) \in \mathcal{S}} \frac{\partial \bar{q}}{\partial z_{p}}\left(\frac{q}{\bar{q}}-1\right) \\
& +\sum_{n=1}^{N} \sum_{(x, y) \in \mathcal{S}}\left(\frac{\partial^{2} \bar{q}}{\partial z_{p}^{2}}\left(\frac{q}{\bar{q}}-1\right)-\left(\frac{\partial \bar{q}}{\partial z_{p}}\right)^{2} \frac{q}{\bar{q}^{2}}\right)\left(z_{p}-\hat{z}_{p}\right) \equiv 0 .
\end{aligned}
$$

Solving (6) for $\hat{z}_{p}$ then leads to the following iterative expression:

$$
\hat{z}_{p}^{(k+1)}=\hat{z}_{p}^{(k)}-\frac{\sum_{n=1}^{N} \sum_{(x, y) \in \mathcal{S}}\left(\frac{\partial \bar{q}}{\partial z_{p}}\left(\frac{q}{\bar{q}}-1\right)\right)}{\sum_{n=1}^{N} \sum_{(x, y) \in \mathcal{S}}\left(\frac{\partial^{2} \bar{q}}{\partial z_{p}^{2}}\left(\frac{q}{\bar{q}}-1\right)-\left(\frac{\partial \bar{q}}{\partial z_{p}}\right)^{2} \frac{q}{\bar{q}^{2}}\right)} .
$$

As the derivation of this result shows, it is necessary to know the position of the focal plane relative to the interface between the coverslip and the specimen (Fig. 1-a). 


\section{PDB1}

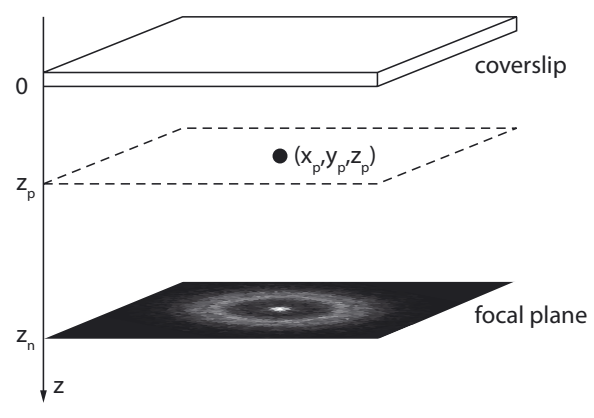

(a)

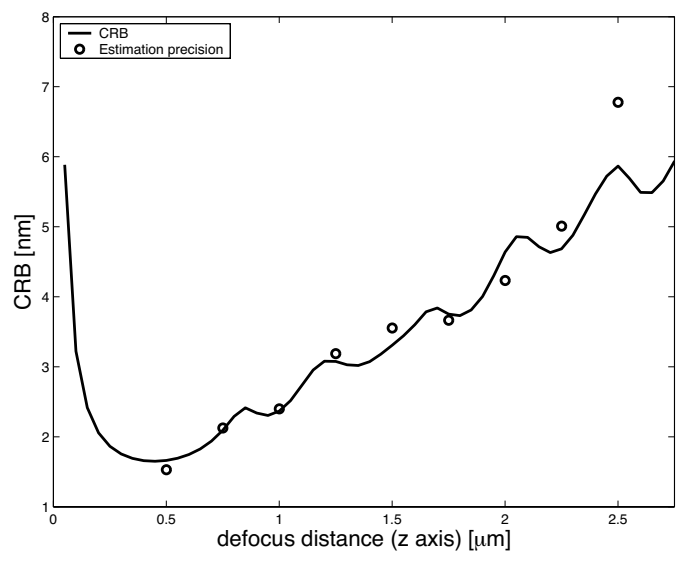

(b)
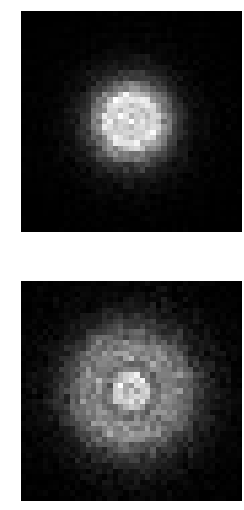

(c)

Fig. 1. (a) Schematic of the setup for one acquisition. (b) Precision of the position estimation with different defocus distances for a particle located directly below the coverslip (for 15 trials per distance). Acquisition parameters: objective: $63 \mathrm{x}$ magnification, $1.25 \mathrm{NA}, \mathrm{CCD}$ pixel size: $6.7 \mu \mathrm{m}$. (c) Typical acquisitions of a particle defocused by $1 \mu \mathrm{m}$ (top) and $1.5 \mu \mathrm{m}$ (bottom)

Finally, in order to obtain an initial estimate for a particle's position, an efficient approach consists in computing the normalized cross-correlation between the acquired image(s) and a small number of sections precomputed from the 3-D PSF:

$$
\hat{z}_{p}^{(0)}=\arg \max _{z_{p}} \frac{\sum_{n=1}^{N} \sum_{(x, y) \in \mathcal{S}}\left(q-\mu_{q}\right)\left(\bar{q}-\mu_{\bar{q}}\right)}{\sqrt{\sum_{n=1}^{N} \sum_{(x, y) \in \mathcal{S}}\left(q-\mu_{q}\right)^{2} \sum_{n=1}^{N} \sum_{(x, y) \in \mathcal{S}}\left(\bar{q}-\mu_{\bar{q}}\right)^{2}}},
$$

where $\mu_{q}$ and $\mu_{\bar{q}}$ are the mean values of pixels in the acquisitions and model, respectively.

\section{Results and conclusion}

Fig. 1-b shows the standard deviation of the precision obtained with our localization method; it closely matches the values predicted by the CRB. These results were generated using the theoretical PSF model described by Gibson and Lanni [1], and show that in the ideal case, it is possible to recover the axial positions of particles with a precision in the nanometer range, even if the acquisition corresponds to particles that are defocused by several micrometers (Fig. 1-c). In addition this result shows that it is most advantageous to image particles off-focus, since their position can then be recovered with greater accuracy. When multiple acquisitions are used, our method can also be used to establish the optimal experimental configuration (i.e., the position of the focus for each acquisition) that guarantees the highest precision for the estimation over a predefined section of the specimen.

\section{References}

[1] S.F. Gibson and F. Lanni. Experimental test of an analytical model of aberration in an oil-immersion objective lens used in three dimensional light microscopy. Journal of the Optical Society of America A, 8(10):1601-1613, 1991.

[2] U. Kubitscheck. Single protein molecules visualized and tracked in the interior of eukaryotic cells. Single Molecules, 3:267-274, 2002.

[3] R.J. Ober, S. Ram, and S. Ward. Localization accuracy in single-molecule microscopy. Biophysical Journal, 86:1185-1200, 2004.

[4] M. Speidel, A. Jonas, and E.-L. Florin. Three-dimensionnal tracking of fluorescent nanoparticles with subnanometer precision by use of off-focus imaging. Optics Letters, 28(2):69-71, 2002. 